\title{
A returning migrant worker with avian influenza A (H7N9) virus infection in Guizhou, China: a case report
}

\author{
Dingming Wang ${ }^{1}$, Guangpeng Tang ${ }^{1}$, Yan Huang ${ }^{1}$, Chun Yu¹, Shijun Li', Li Zhuang ${ }^{1}$, Lin Fu' ${ }^{1}$ Shiping Wang ${ }^{2}$,
} Nanshi Li ${ }^{3}$, Xiyan Li $i^{4}$ Lei Yang ${ }^{4}$, Yu Lan ${ }^{4}$, Tian Bai ${ }^{4}$ and Yuelong Shu ${ }^{4^{*}}$

\begin{abstract}
Introduction: Human infection with avian influenza A (H7N9) virus was first reported on March, 2013 in the Yangtze River Delta region of China. The majority of human cases were detected in mainland China; other regions out of mainland China reported imported human cases, including Hong Kong SAR, Taiwan (the Republic of China) and Malaysia, due to human transportation. Here, we report the first human case of H7N9 infection imported into Guizhou Province during the Spring Festival travel season in January 2014.
\end{abstract}

Case presentation: In early January 2014, a 38-year-old healthy Chinese man, a migrant worker returning from previously H7N9-affected Zhejiang Province, was identified as the first human case of infection with avian influenza A(H7N9) virus in Guizhou Province. He developed fever in Zhejiang at the beginning of January 2014, and returned to Guizhou for the Chinese New Year the next day. He went to seek medical care, but deteriorated rapidly and died on day 8 after his illness onset. The influenza virus A/Guizhou/01502/2014 isolated from the patient had 99\% identity with viruses circulating in the Yangtze River Delta region. Selected amino acids substitutions, well-known to be associated with mammalian adaptation, viral replication and drug resistance were similar to other H7N9 viruses circulating in humans.

Conclusions: Epidemiology investigation and laboratory results confirmed it was the first imported case of H7N9 infection in Guizhou Province. This finding further indicated that more human H7N9 cases may be detected in other regions due to frequent travel both domestically and internationally.

Keywords: H7N9 virus, Influenza, Migrant worker, Imported case, Epidemiology investigation

\section{Introduction}

The first wave of human infections with avian influenza A(H7N9) virus began in spring and subsided by summer 2013 in China [1], and it has re-emerged since October 2013 [2]. As of March 10, 2014, a total of 379 cases and 135 deaths were reported in mainland China [3]. However, human cases were also detected in Hong Kong SAR, Taiwan (the Republic of China) [4] and recently the Malaysian Ministry of Health announced the first imported case outside of China on February 12, 2014 [5]. It indicated that human cases could be exported to

\footnotetext{
* Correspondence: yshu@cnic.org.cn

${ }^{4}$ National Institute for Viral Disease Control and Prevention, Chinese Center for Disease Control and Prevention, Key Laboratory for Medical Virology, National Health and Family Planning Commission, 155 Changbai Road, Beijing 102206, P. R China

Full list of author information is available at the end of the article
}

other regions by frequent travel both domestically and internationally. Each year, billions of people migrate for the Spring Festival in China, therefore increasing the risk of infectious diseases spreading. Here we report the first human case of H7N9 infection imported to Guizhou Province from the epidemic region during the period of the so-called largest annual human migration.

\section{Case presentation}

The patient was a 38-year-old healthy Chinese man, a migrant worker who worked in Zhejiang Province. As shown in Figure 1, he developed influenza-like illness (ILI) symptoms, including headache and $40^{\circ} \mathrm{C}$ fever at the beginning of January 2014, and sought medical attention in a village clinic and received infusion therapy. He went back to his hometown in Guizhou Province for 


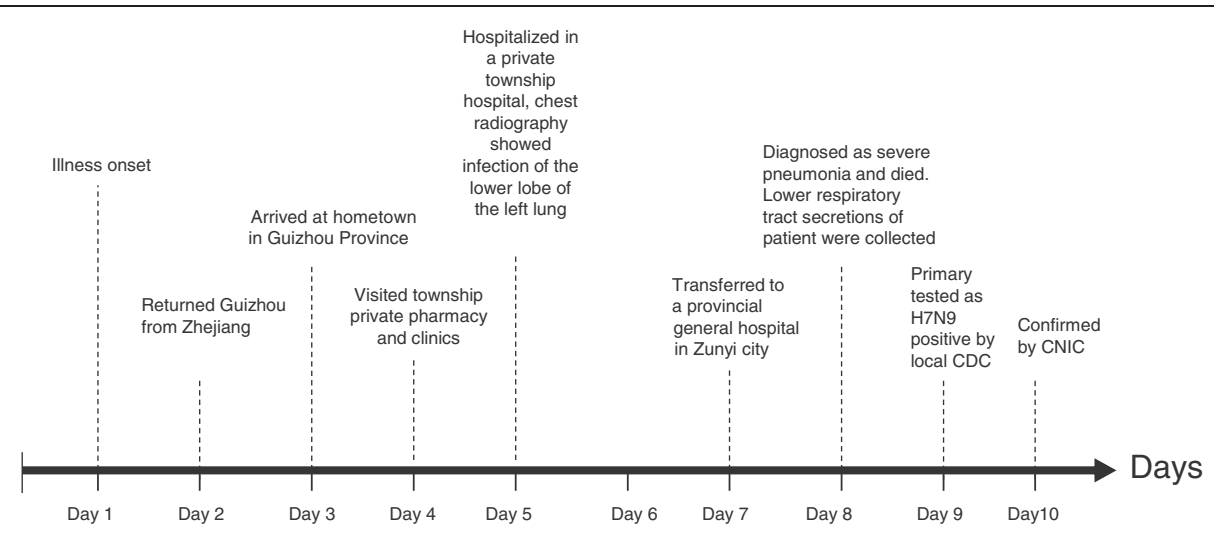

Figure 1 Timeline of potential exposure and medical consultation of a laboratory-confirmed imported influenza A(H7N9) case, Guizhou Province, China, January 2014.

the coming Spring Festival by taking a long-distance bus from Zhejiang Province one day after he developed fever. He then sought medical care in a village clinic and a township hospital when he arrived at his hometown. His chest radiograph showed pneumonia in the lower lobe of his left lung (the CT value ranged from -12 to approximately -19) and he was hospitalized in a township hospital on day 5 after his illness onset. He experienced high fever and rapid progression, and was transferred to a provincial general hospital in Zunyi city. $\mathrm{He}$ was diagnosed as severe pneumonia, deteriorated rapidly and died on day 8 after his illness onset. He had received symptomatic treatment during the clinical treatment and all drugs that had been used in the township hospital and the general hospital in Zunyi city are listed in Table 1. No antiviral drugs were administrated during the clinical treatment. The case was reported through the Chinese surveillance system for pneumonia with unknown etiology and confirmed as human infection with avian influenza $\mathrm{A}(\mathrm{H} 7 \mathrm{~N} 9)$ virus by means of RT-PCR, virus isolation and full genome sequencing at the Chinese National Influenza Center (CNIC). Since the patient had died, written informed consent was obtained from the patient's next-of-kin for publication of this case report.

Table 1 Drugs used during clinical treatment in hospital

\begin{tabular}{ll}
\hline Days after illness onset & Treatment \\
\hline Day 5 & Antibiotic treatment with vancomycin+ \\
& ceftazidime+aminoglycoside \\
& Use of omeprazole to prevent stress ulcer \\
& Use of dexamethasone to reduce \\
& inflammatory exudation \\
& Meropenem \\
Day 7 & Compound aminopyrine and acetaminophen \\
& Ceftriaxone, bromhexine, doxofylline and \\
& coenzyme complex \\
\hline
\end{tabular}

Sixty-one close contacts were identified by epidemiological survey, including 36 health-care personnel, eight medical escorts and 17 households and family contacts. As a public health response, written informed consents from contacts can be waived according to Chinese law. The close contacts were medically monitored for seven consecutive days; no ILI symptoms were observed in all contacts except for the patient's family members. One family member appeared with a sore throat and developed a $38^{\circ} \mathrm{C}$ fever five to seven days after the patient's illness onset, and another developed a sore throat without fever seven days after the patient's illness onset. Continuous throat swabs were collected from them; all the samples were negative for H7N9 influenza virus. Antibody response to H7N9 virus was not detected from the paired sera of all contacts.

The influenza virus A/Guizhou/01502/2014 was isolated from the patient. The PB2 gene had a high degree of similarity (99\%) to viruses isolated from human in Zhejiang Province. Another seven segments showed the highest identity with viruses isolated from birds in the Yangtze River Delta region, where Zhejiang Province is located (Table 2). The phylogenetic analysis further demonstrated all eight gene segments were clustered with

Table 2 The highest homology of the virus isolated from the patient with other H7N9 viruses

\begin{tabular}{lll}
\hline Segment & Strain & Identity \\
\hline PB2 & A/Zhejiang/LS01/2014 (H7N9) & 2272/2280 (99\%) \\
PB1 & A/chicken/Suzhou/040201H/2013 (H7N9) & 2265/2274 (99\%) \\
PA & A/duck/Sunan/040802G/2013(H7N9) & 2145/2151 (99\%) \\
HA & A/chicken/Suzhou/097-1/2013(H7N9) & $1668 / 1683(99 \%)$ \\
NP & A/tree_sparrow/Shanghai/01/2013 (H7N9) & $1493 / 1497(99 \%)$ \\
NA & A/chicken/Suzhou/040201H/2013 (H7N9) & $1389 / 1398(99 \%)$ \\
MP & A/chicken/Suzhou/097-1/2013 (H7N9) & $981 / 982(99 \%)$ \\
NS & A/pigeon/Wuxi/0405007G/2013(H7N9) & $836 / 838(99 \%)$ \\
\hline
\end{tabular}


viruses that circulated in this region (Figures 2 and 3). A/Shanghai/1/2013 (SH1, the first H7N9 virus isolate), A/Anhui/1/2013 (AH1, the vaccine strain recommended by the World Health Organization) and those six virus isolates with highest identity with A/Guizhou/01502/ 2014 mentioned in Table 2 were selected for characteristic comparison of the critical amino acids residues (Table 3). Similar to those viruses, the mammalianadaptive mutations such as G186V and Q226L in the HA gene that were previously reported [6] were found in this virus. It remained $R$ in the position 292 of the
NA gene and suggested the effectiveness of oseltamivir antiviral treatment. The virus possessed a $627 \mathrm{~K}$ mutation in the PB2 gene, which was proposed to be associated with high polymerase activity and increased the virulence in mice [7]. The PB1 L368V mutation was found in this virus, which increased transmission in ferret [8]. The signature amino acids of the human influenza virus in the PA gene were detected, including V100A, K356R and S409N [9,10]; however, a unique mutation L336M in the PA gene that had only been reported in a Hong Kong isolate [11] was not detected.

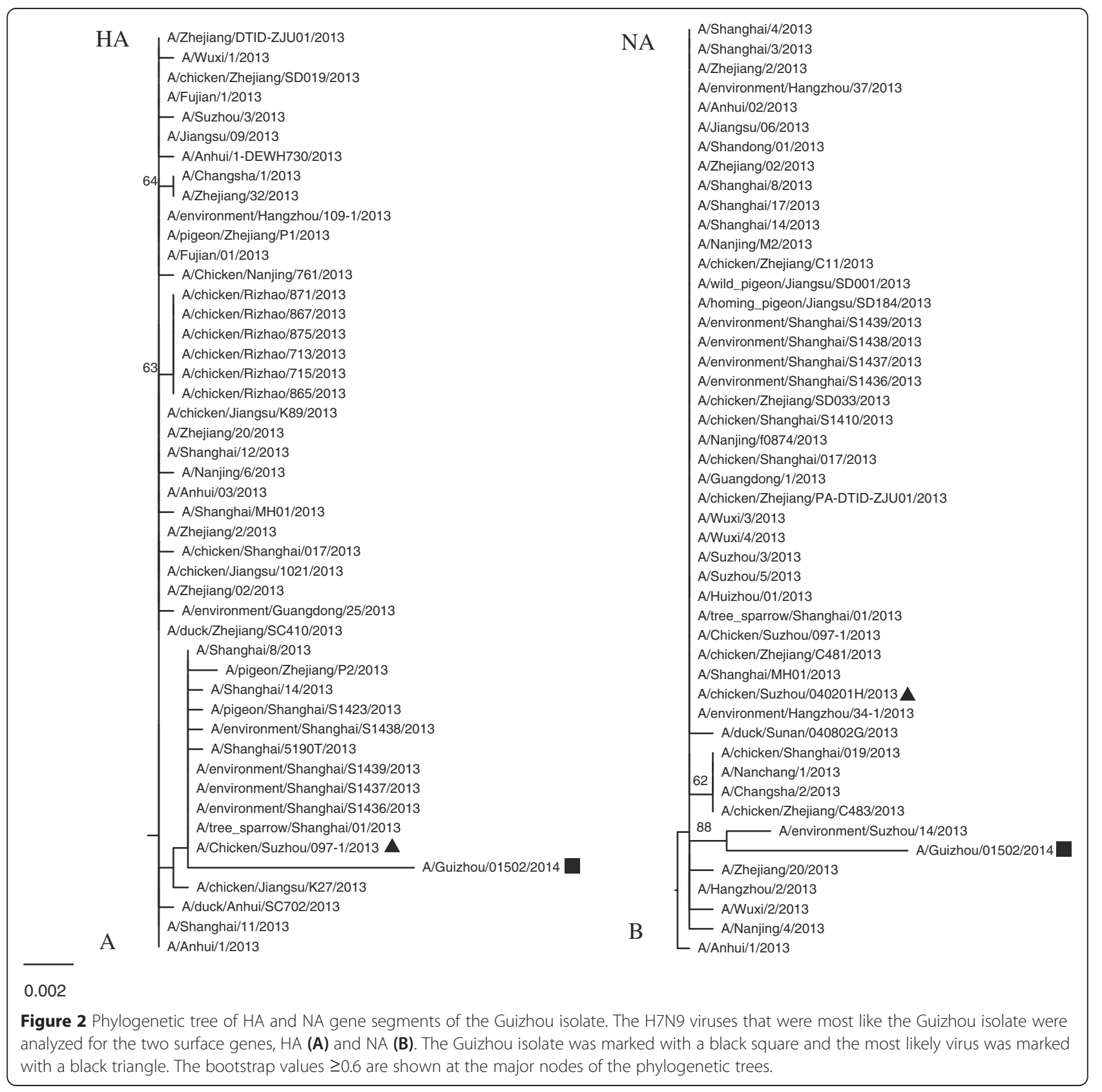




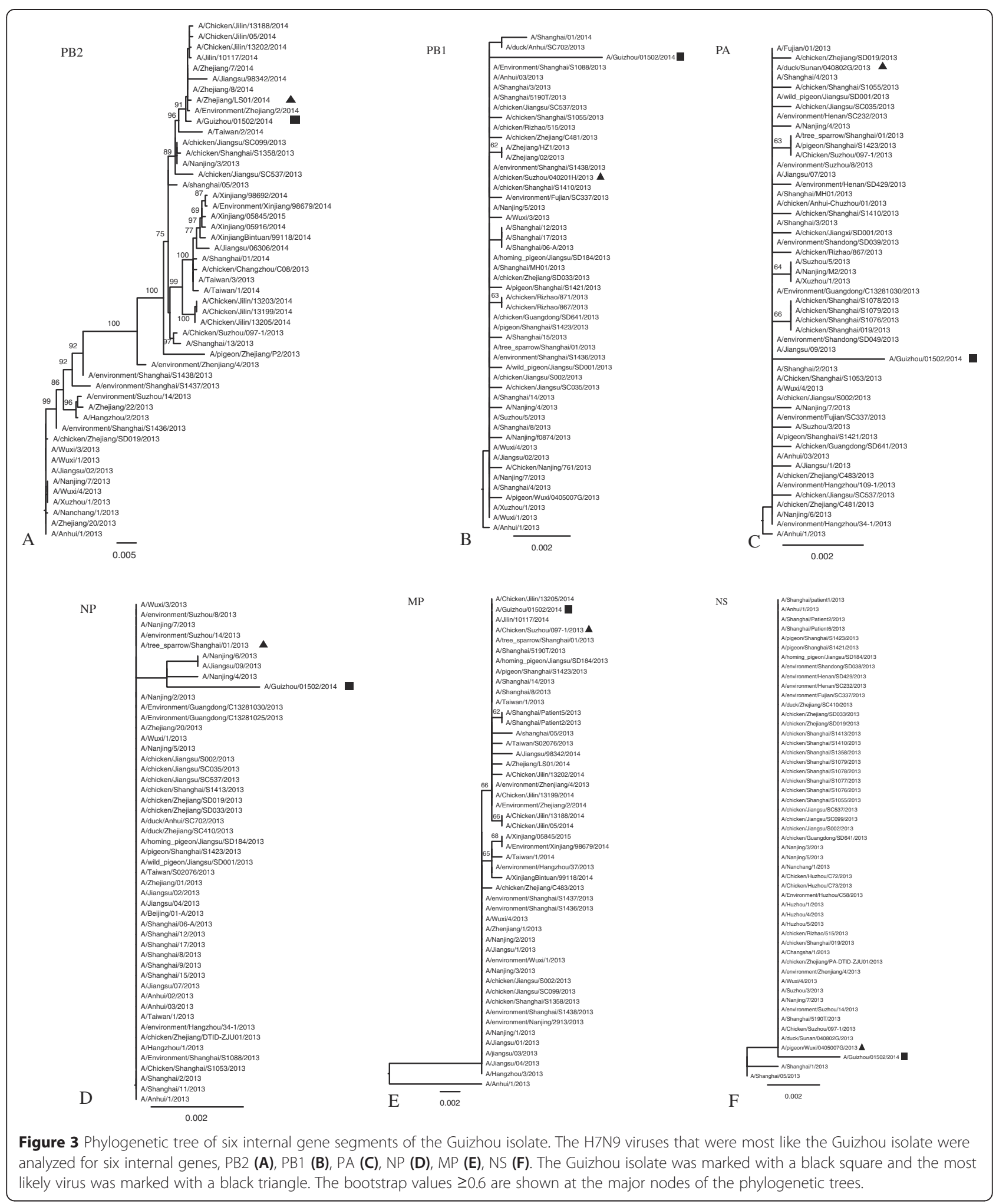

\section{Conclusions}

This is the first imported human H7N9 case of a migrant worker moving from epidemic areas to unaffected regions during the Spring Festival migration, which is a period of travel in China with an extremely high traffic load around the time of the Chinese New Year.

Unlike most previously reported fatal cases of patients who were older and/or who had underlying medical 


\section{Table 3 Comparison of selected molecular markers between A/Guizhou/01502/2014 and representative H7N9 viruses}

\begin{tabular}{|c|c|c|c|c|c|c|c|c|c|c|c|}
\hline Protein & Phenotypic consequences $^{\mathrm{a}}$ & $\begin{array}{l}\text { Amino acid } \\
\text { position } / \text { motif }^{b}\end{array}$ & GZ 01502 & SH1 & AH1 & ZJLS01 & SZ040201H & SN040802G & CK/SZ097-1 & tree_sparrow/SH1 & pigeon/WX/0405007G \\
\hline \multirow[t]{2}{*}{ HA } & \multirow{2}{*}{$\begin{array}{l}\text { Increased virus binding to human-type } \\
\text { receptors (a2-6) }\end{array}$} & G186V & V & G & V & V & V & V & V & V & V \\
\hline & & Q226L & L & Q & L & L & L & Q & L & L & Q \\
\hline \multirow[t]{2}{*}{ NA } & $\begin{array}{l}\text { Enhanced virulence in mice/adaption and } \\
\text { transmission in domestic poultry }\end{array}$ & $\begin{array}{l}\text { 69-73 amino } \\
\text { acids deletion }\end{array}$ & Yes & Yes & Yes & Yes & Yes & Yes & Yes & Yes & Yes \\
\hline & Related to drug resistance & R292K & $\mathrm{R}$ & K & $\mathrm{R}$ & K & $\mathrm{R}$ & $\mathrm{R}$ & $\mathrm{R}$ & $\mathrm{R}$ & $\mathrm{R}$ \\
\hline \multirow[t]{2}{*}{ PB2 } & Increased virulence in mice & E627K & k & K & K & K & $E$ & $E$ & $E$ & K & E \\
\hline & $\begin{array}{l}\text { Enhanced virulence and transmission in } \\
\text { guinea pigs }\end{array}$ & D701N & $\mathrm{D}$ & D & $\mathrm{D}$ & $\mathrm{D}$ & $\mathrm{D}$ & $\mathrm{D}$ & $\mathrm{D}$ & $\mathrm{D}$ & $\mathrm{D}$ \\
\hline \multirow[t]{2}{*}{ PB1 } & Increased transmission in ferret & I368V & V & । & V & V & V & V & V & V & V \\
\hline & Increased replication in mammalian cells & L598P & L & M & $L$ & L & L & L & L & L & L \\
\hline PB1-F2 & Increased pathogenicity in mice & $\begin{array}{l}87-90 \text { amino } \\
\text { acids in length }\end{array}$ & 90 & 90 & 90 & 90 & 90 & 90 & 25 & 90 & 90 \\
\hline \multirow[t]{4}{*}{ PA } & Increased polymerase activity & L336M & L & L & L & L & L & L & L & L & L \\
\hline & Species-associated signature positions & V100A & A & $A$ & $A$ & $A$ & $A$ & $A$ & $A$ & $A$ & A \\
\hline & & K356R & $R$ & $R$ & $R$ & $R$ & $R$ & $R$ & $R$ & $R$ & $R$ \\
\hline & & S409N & $N$ & N & $N$ & $N$ & $N$ & $N$ & $N$ & $N$ & $N$ \\
\hline
\end{tabular}

The mutation to the right of the amino acid position confers the phenotype described below.

${ }^{\mathrm{b}}$ The HA gene was under the H3 numbering system. The NA gene was under the N2 numbering system. Other internal genes were numbered from the start codon (Met). All letters in italics represent mutations detected at the relative position. 
conditions [12], this patient was young and reported without any underlying medical conditions. No mutations associated with antiviral resistance were detected; one possibility for the rapid deterioration and death of the patient may be due to the fact antiviral drugs were not used during his treatment. The virus isolated from the patient showed a high identity with viruses circulating in the Yangtze River Delta region and he developed respiratory syndromes before his arrival in Guizhou Province. He visited a neighborhood live poultry market before his illness onset, which indicated the potential infection source was likely to be the infected poultry in the market, as previously reported elsewhere [12-14]. Further, the H7N9 virus was not detected in Guizhou based on the results of poultry-related environmental surveillance since 2009 (data not shown). These data concluded that the case had been introduced from an affected region by the movement of people. Imported human cases of the H7N9 virus travelling from affected areas to non-endemic areas domestically and internationally have been reported in Hong Kong SAR, Taiwan (the Republic of China) and Malaysia during the first and second wave of H7N9 infections $[4,5,15]$. In each year, millions of migrant workers from rural areas of Guizhou Province go to work in the Yangtze River Delta and the Pearl River Delta and return home for a reunion with their family. With the increasingly frequent movement of people, it is foreseen that more human H7N9 cases may be detected in other regions due to frequent travel both domestically and internationally. Considering the rapidly progressive pneumonia, acute respiratory distress syndrome (ARDS), the high rates of ICU admission and death, early detection and antiviral drug therapy of suspected cases imported from affected areas are essential to reduce the case fatality rate of H7N9 virus infection.

The undetected H7N9 virus of the respiratory specimens and the negative antibody response to H7N9 in his family members who presented clinical syndromes indicated that the transmission from the patient to his family members could be ruled out.

\section{Consent}

Written informed consent was obtained from the patient's next-of-kin for publication of this case report and any accompanying images. A copy of the written consent is available for review by the Editor-in-Chief of this journal.

\section{Competing interests}

The authors declare that they have no competing interests. testing. $L Z, L F, S P W, X Y L$ and $Y L$ participated in sequencing and virus isolation. $Y L$ participated in the sequence alignment, and analyzed the laboratory data. TB was a major contributor in writing the manuscript. YLS conceived and coordinated the study. All authors read and approved the final manuscript.

\section{Acknowledgements}

We thank our other colleagues who participated in the field investigation for the epidemiology survey. This study was supported by the China National Mega-projects for Infectious Diseases (2014ZX10004002).

\section{Author details}

${ }^{1}$ Guizhou Provincial Center for Disease Control and Prevention, 101 Bageyan Road, Guiyang City 550004, Guizhou Province, China. ${ }^{2}$ Zunyi Prefecture Center for Disease Control and Prevention, 19 Xima Road, Zunyi City 563002, Guizhou Province, China. ${ }^{3}$ Meitan County Center for Disease Control and Prevention, 99-8 Zhedanan Road, Meitan County 564100, Guizhou Province, China. ${ }^{4}$ National Institute for Viral Disease Control and Prevention, Chinese Center for Disease Control and Prevention, Key Laboratory for Medical Virology, National Health and Family Planning Commission, 155 Changbai Road, Beijing 102206, P. R China.

Received: 20 September 2014 Accepted: 26 March 2015

Published online: 12 May 2015

\section{References}

1. Gao R, Cao B, Hu Y, Feng Z, Wang D, Hu W, et al. Human infection with a novel avian-origin influenza A (H7N9) virus. N Engl J Med. 2013;368:1888-97.

2. Chen $E$, Chen Y, Fu L, Chen Z, Gong Z, Mao H, et al. Human infection with avian influenza A(H7N9) virus re-emerges in China in winter 2013. Euro Surveill. 2013;18:20616.

3. Chinese National Influenza Center. Weekly report. 2014. http://www.cnic.org.cn/ chn/down/showdown.php?downid=799.

4. Chang SY, Lin PH, Tsai JC, Hung CC, Chang SC. The first case of H7N9 influenza in Taiwan. Lancet. 2013;381:1621.

5. World Health Organization. Human infection with avian influenza A(H7N9) virus - update, Disease outbreak news. 2014. www.who.int/entity/influenza/ human_animal_interface/avian_influenza/archive/en.

6. Dortmans JC, Dekkers J, Wickramasinghe IN, Verheije MH, Rottier PJ, van Kuppeveld FJ, et al. Adaptation of novel H7N9 influenza A virus to human receptors. Sci Rep. 2013;3:3058.

7. Zhang H, Li X, Guo J, Li L, Chang C, Li Y, et al. The PB2 E627K mutation contributes to the high polymerase activity and enhanced replication of H7N9 influenza virus. J Gen Virol. 2014;95:779-86.

8. Herfst S, Schrauwen EJ, Linster M, Chutinimitkul S, de Wit E, Munster VJ, et al. Airborne transmission of influenza $\mathrm{A} / \mathrm{H} 5 \mathrm{~N} 1$ virus between ferrets. Science. 2012;336:1534-41.

9. Taubenberger JK, Reid AH, Lourens RM, Wang R, Jin G, Fanning TG. Characterization of the 1918 influenza virus polymerase genes. Nature. 2005;437:889-93.

10. Chen GW, Lai MM, Wu SC, Chang SC, Huang LM, Shih SR. Is avian influenza A (H7N9) virus staggering its way to humans? J Formos Med Assoc. 2013;112:312-8.

11. To KK, Song W, Lau SY, Que TL, Lung DC, Hung IF, et al. Unique reassortant of influenza A (H7N9) virus associated with severe disease emerging in Hong Kong. J Infect. 2014;69:60-8.

12. Li Q, Zhou L, Zhou M, Chen Z, Li F, Wu H, et al. Preliminary report: epidemiology of the avian influenza A(H7N9) outbreak in China. N Engl J Med. 2013. doi:10.1056/NEJMoa1304617.

13. Liu D, Shi W, Wang D, Xiao H, Li W, Bi Y, et al. Origin and diversity of novel avian influenza A H7N9 viruses causing human infection: phylogenetic, structural, and coalescent analyses. Lancet. 2013;381:1926-32.

14. Shi JDG, Liu P. Isolation and characterization of H7N9 viruses from live poultry markets-Implication of the source of current H7N9 infection in humans. Chinese Sci Bull. 2013;58:1857-63.

15. The Centre for Health Protection. Statutory notification of novel influenza A infection. 2014. http://www.chp.gov.hk/en/content/568/33467.html.

\section{Authors' contributions}

DMW carried out the field investigation, participated in laboratory testing, and drafted the manuscript. GPT, YH, CY, NSL and LY participated in the field investigation. SJL interpreted and analyzed the surveillance and RT-PCR 